\title{
Dynamic Response due to Breaking Wave Impact Force on an Offshore Wind Turbine Support Structure*
}

\author{
Kenji SHIMADA** and Takeshi ISHIHARA*** \\ **Institute of Technology, Shimizu Corporation, \\ 3-4-17 Etchujima, Koto-ku, 135-8530 Japan \\ E-mail: shimaken@shimz.co.jp \\ *** Department of Civil Engineering, School of Engineering, The University of Tokyo, \\ 7-3-1, Hongou, Bunkyo-ku, Tokyo 113-8656, Japan
}

\begin{abstract}
Dynamic response due to breaking wave impact force on an offshore wind turbine support structure is investigated, especially the effects of higher modes. From comparison between two representative breaking wave impact force models, i.e. the Goda model and the Wienke model, it is clarified that their impulse response functions in the low-frequency region and high-frequency region reverse their magnitudes. For convenience in the primitive design stage, a procedure for prediction of the dynamic response by a breaking wave impact force acting on a support structure is proposed using the impulse response function. By taking only a few vibration modes, the maximum values of the section forces on the support structure can be estimated without any cumbersome time dependent analyses. Furthermore this paper proposes an approximation of the curling factor in terms of the surf similarity parameter.
\end{abstract}

Key words: Offshore Wind Turbine, Impact Breaking Wave Force, Modal Analysis

\section{Introduction}

For a fixed type offshore wind turbine built in a surf zone, it is necessary to consider the impact force due to breaking waves on its pile support structure for its structural safety. Wave force is assumed to consist of non-breaking wave force and breaking wave impact force. For loading on a stiff pile, for example, Goda ${ }^{(1)}$ has proposed a quasi static approach incorporating nonlinearities in regular wave dynamics. He has also proposed an effective breaking wave impact load, which provides a convenient way of estimating the maximum section load that does not require any cumbersome time dependent analyses. By combining both of these methods, a convenient design method for predicting section loads arising in a support structure of an offshore wind turbine tower can be established, especially in the primitive stage of structural design.

Since the actual structure of a wind turbine possesses multiple eigen values, not only the fundamental vibration mode but also higher mode responses can be excited. However, the Goda method ${ }^{(2)}$ does not deal with these higher modes. In this paper, Goda's effective breaking wave impact load is extended to higher vibration mode. 


\section{Formulations of Secton Forces by Breaking Wave Impact Force}

It is well known that the wave breaking impact force is well accounted by the added mass theory. Goda ${ }^{(2)}$ has formulated breaking wave impact force based on von Karman's formulation. On the other hand, Wienke et al. ${ }^{(3)(4)}$ have formulated it according to Wagner's formulation, which modifies the von Karman's theory by taking into account pile-up effect. These breaking wave impact forces are expressed as :

$$
F_{I}(\tau)=\lambda \eta_{c} \pi \rho R C_{b}^{2} C_{s}(\tau)
$$

where $\tau$ is reduced time $=t / t_{B}, t$ is time $(\mathrm{s}), t_{B}=R / C . C_{b}$ is wave celerity $(\mathrm{m} / \mathrm{s})$ and $R$ is radius of a support pile $(\mathrm{m})$. It should be noted that $t_{B}$ corresponds to impulse force duration in the Goda model, whereas in Wienke's model it is $(13 / 32) t_{B}$. In the following studies, $t_{B}$ is used for time normalization of both models. $\rho$ is density of sea water $\left(\mathrm{kg} / \mathrm{m}^{3}\right), \lambda$ is curling factor and $\eta_{c}$ is water surface elevation (m), see Fig.3. $C_{s}(\tau)$ is a temporal slamming effect and has been proposed as :

Goda model:

$$
C_{s}(\tau)=1-\tau
$$

Wienke model:

$$
\begin{aligned}
& C_{s}(\tau)=2\left(1-\frac{1}{\pi} \sqrt{\tau} \tanh ^{-1} \sqrt{1-\frac{\tau}{4}}\right),\left(0 \leq \tau \leq \frac{1}{8}\right) \\
& C_{s}(\tau)=\sqrt{\frac{1}{6 \tau_{2}}}-\frac{1}{\pi} \sqrt[4]{\frac{8}{3} \tau_{2}} \tanh ^{-1} \sqrt{1-\tau_{2} \sqrt{6 \tau_{2}}},\left(\frac{3}{32}<\tau_{2} \leq \frac{3}{8}, \tau_{2}=\tau-\frac{1}{32}\right)
\end{aligned}
$$

Here, since equations (1) to (3) are time dependent, if time dependent analysis is performed, section forces acting on a support structure of a wind turbine are obtained. However, in the following, Goda's effective breaking wave impulsive force ${ }^{(2)}$ is extended to a higher vibration mode by the modal analysis. The equation of motion for the $j$-th mode is expressed as :

$$
\frac{d^{2} X_{j}}{d \tau^{2}}+\Omega_{j}^{2} X_{j}=\Omega_{j}^{2} C_{s}(\tau), \Omega_{j}=\omega_{j} t_{B}
$$

where $\omega_{j}=2 \pi f_{j}$ is circular frequency, $f_{j}$ is the $j$-th natural frequency of the structure of the wind turbine, $X_{j}=x_{j} / x_{0}, x_{0}=F_{I_{j 0}} / k_{j}, x_{j}$ is the $j$-th generalized displacement, $k_{j}$ is the $j$-th generalized stiffness and $F_{I_{j 0}}$ is the effective breaking wave impact force of the $j$-th mode which is obtained as :

$$
F_{I_{j 0}}=\pi \rho C_{b}^{2} R \int_{h+(1-\lambda) \eta_{c}}^{h+\eta_{c}} \phi_{j}(z) d z
$$

where, $h$ is water depth and $\phi_{j}(z)$ is the $j$-th mode value at height $z$ measured from the sea bed. $F_{I_{j 0}}$ corresponds to the initial value of the $j$-th generalized breaking wave impact force. The effective breaking wave impact force of the $j$-th mode is expressed as :

$$
\hat{F}_{I_{j}}=F_{I_{j 0}} \hat{X}_{j}
$$

$\hat{X}_{j}$ is the impulse response factor of the $j$-th mode, which is defined as the maximum 
response of the Eq.(4), and is expressed as :

Goda model :

$$
\hat{X}_{j}=\left\{\begin{array}{cc}
\sqrt{\left(1-\frac{1}{\Omega_{j}} \sin \Omega_{j}\right)^{2}+\frac{1}{\Omega_{j}^{2}}\left(1-\cos \Omega_{j}\right)^{2}} & \cdots \Omega_{j}<2.33 \\
2-\frac{2}{\Omega_{j}} \tan ^{-1} \Omega_{j} & \cdots \Omega_{j} \geq 2.33
\end{array}\right.
$$

Wienke model :

$$
\hat{X}_{j}= \begin{cases}\sum_{n=1}^{3} c_{n} \Omega_{j}^{n} & \cdots \Omega_{j} \leq 19 \\ 2.9674\left(\Omega_{j}-18\right)^{0.033} & \cdots 19<\Omega_{j} \leq 2000\end{cases}
$$

where, $c_{1}=0.38, c_{2}=-0.019$ and $c_{3}=0.00038$. As can be found in Eq.(3), since the Wienke model is a complicated function of time, impulse response factor in Eq.(8) was obtained by fitting the result calculated by time dependent analysis by the Newmark $\beta$ method.

The maximum section forces are obtained by combining an appropriate number of modal components. For superposition of the modal components, the Complete Quadratic Combination Method (CQC method) and the Square Root of Sum of Squares Method (SRSS method) are frequently used. The CQC method considers the effect of damping, but in a problem involving impact force the damping is sometimes neglected since the maximum response occurs at the first or second peak. Here, as the simplest approximation, the SRSS method is used. The maximum shear and bending moment of the $i$-th level $\hat{Q}_{i}$ and $\hat{M}_{i}$ can be obtained by SRSS method as :

$$
\begin{aligned}
& \hat{Q}_{I_{i}}=\sqrt{\sum_{j=1}^{n} \hat{Q}_{i j}^{2}} \\
& \hat{M}_{I_{i}}=\sqrt{\sum_{j=1}^{n} \hat{M}_{i j}^{2}} \\
& \hat{\mathbf{M}}_{j}=\left[\begin{array}{cccc}
\Delta z_{N} & 0 & \cdots & 0 \\
\Delta z_{N} & \Delta z_{N-1} & \cdots & 0 \\
\vdots & \vdots & \ddots & \vdots \\
\Delta z_{N} & \Delta z_{N-1} & \cdots & \Delta z_{1}
\end{array}\right] \hat{\mathbf{Q}}_{j} \\
& \hat{\mathbf{Q}}_{j}=\left[\begin{array}{cccc}
1 & 0 & \cdots & 0 \\
1 & 1 & \cdots & 0 \\
\vdots & \vdots & \ddots & \vdots \\
1 & 1 & \cdots & 1
\end{array}\right] \hat{\mathbf{P}}_{j} \\
& \hat{\mathbf{P}}_{j}=[K] \boldsymbol{\varphi}_{j} \hat{x}_{j} \\
& \hat{x}_{j}=\frac{\hat{F}_{I j}}{m_{j} \omega_{j}^{2}} \\
& m_{j}=\boldsymbol{\varphi}_{j}^{T}[M] \boldsymbol{\varphi}_{j}
\end{aligned}
$$

where $[M]$ is a mass matrix, $[K]$ is a stiffness matrix, $\Delta z_{i}=z_{i}-z_{i-1}$ and $\hat{\mathbf{Q}}_{j}, \hat{\mathbf{M}}_{j}, \hat{\mathbf{P}}_{j}, \boldsymbol{\varphi}_{j}$ are maximum shear force vector, maximum moment vector, maximum 
reaction force vector and mode vector. $\hat{x}_{j}$ and $m_{j}$ are generalized displacement and generalized mass of the $j$-th mode. $n$ is the number of the highest mode to be considered.

\section{Results and Discussion}

\subsection{Differences between Goda model and Wienke model}

In Fig.1, the impulse response factors expressed by Eq.(7) and (8) are demonstrated. The abscissa represents the reduced frequency, which is the ratio of impulse force duration to the natural period of each vibration mode, as can be seen in Eq.(4). Both of the models intersect at $\Omega_{j} \approx 4.72$. For $\Omega_{j}<4.72$, the Goda model is larger and for $\Omega_{j}>4.72$ the Wienke model gives larger response estimates. Furthermore, when $\Omega_{j} \rightarrow \infty$, the Goda model approaches to $\hat{X}_{j}=2$ and the Wienke model approaches $\hat{X}_{j}=4$. This corresponds to a ratio of $C_{s}(0)$ of both models of 2 .

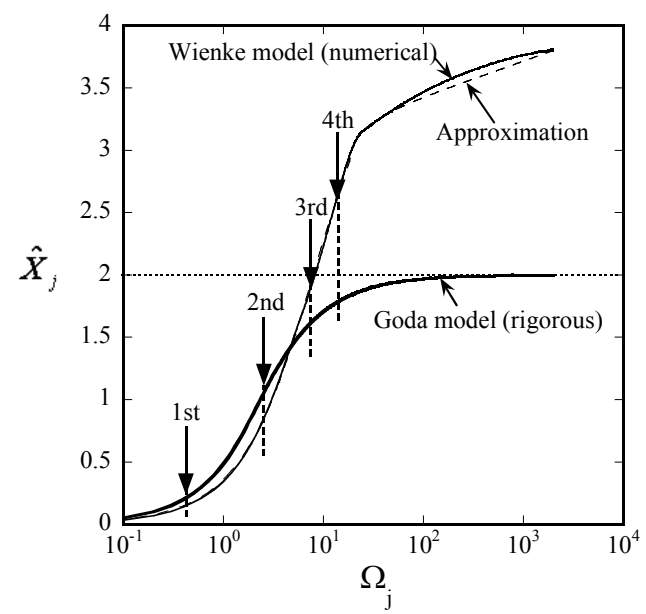

Fig. 1 Impulse response factor for a circular cylinder

Figure 2 shows a model of an offshore wind turbine ${ }^{(5)}$. It is a $6 \mathrm{MW}$ turbine with a twin blade system. Its support structure is assumed to be made of reinforced concrete.

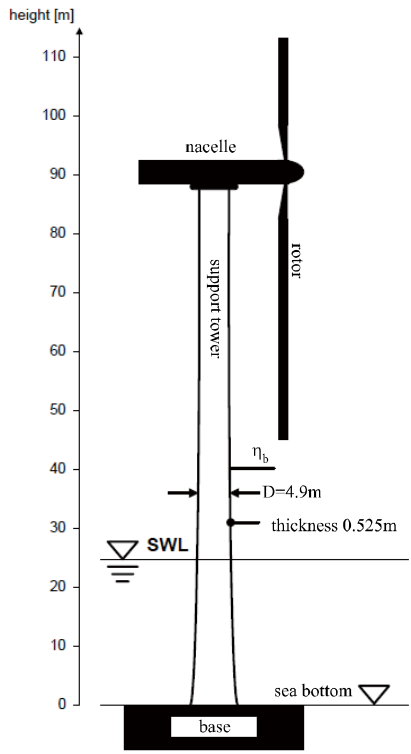

$\begin{array}{lr}\text { Characteristic data } \\ \text { Wind turbine } \\ \text { Power } & \\ \text { Number of blades } & 6 \mathrm{MW} \\ \text { Diameter of rotor } & 2 \\ \text { Support tower } & 100 \mathrm{~m} \\ \text { Material } & \\ \text { Length } & \text { Concrete } \\ \text { Thickness } & 88 \mathrm{~m} \\ \text { Frequency (1st order) } & 0.525 \mathrm{~m} \\ \text { Frequency (2nd order) } & 0.3473 \mathrm{~Hz} \\ \text { Ambient conditions } & 1.941 \mathrm{~Hz} \\ \text { Water depth } & \\ \text { Breaking wave parameters } & 25 \mathrm{~m} \\ \text { Type } & \text { Plunging } \\ \text { Period } & 9 \mathrm{~s} \\ \text { Celerity } & 12.45 \mathrm{~m} / \mathrm{s} \\ \text { Height } & 16 \mathrm{~m} \\ \text { Wave surface elevation } & 15.2 \mathrm{~m} \\ \text { Horizontal asymmetry } & 0.95\end{array}$

Fig. 2 Model of a wind turbine ${ }^{(5)}$ 
Figure 3 shows the analytical model. The whole structure is discretized by ten lumped masses with sway and rotational degrees of freedom for each mass. The mass and stiffness are estimated based on the literature ${ }^{(5)}$ as listed in Fig.3. The added mass is not included since they are limited in the lower part of the structure. Structural damping is provided by Rayleigh damping. Eigen values are listed in Table 1 together with description in the literature $^{(5)}$. The oceanographic condition is also based on the literature: wave height is $16 \mathrm{~m}$, wave period is $9 \mathrm{~s}$ and sea bed depth is $25 \mathrm{~m}$. Although the water surface elevation is represented as $\eta_{c}=15.2 \mathrm{~m}$ in Fig.2, the following results are calculated using $\lambda \eta_{c}=1 \mathrm{~m}$ following the calculation in the literature.

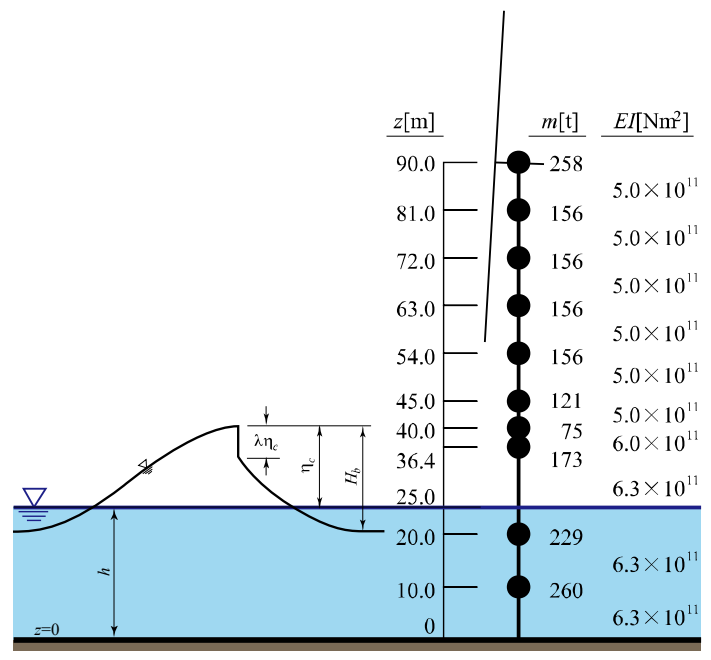

Fig. 3 Analytical model

Table 1. Natural periods

\begin{tabular}{|c|c|c|c|}
\hline \multirow{2}{*}{ Mode $\mathrm{j}$} & Wienke $^{(5)}$ & \multicolumn{2}{|c|}{ Present cal. } \\
\cline { 2 - 4 } & Natural period(s) & Natural period(s) & $\Omega_{\mathrm{j}}$ \\
\hline 1 & 2.879 & 2.962 & 0.417 \\
\hline 2 & 0.515 & 0.487 & 2.538 \\
\hline 3 & - & 0.167 & 7.411 \\
\hline 4 & - & 0.092 & 13.46 \\
\hline
\end{tabular}

The calculated time series of the base bending moment is presented in Fig.4. Here, structural damping is assumed to be $\zeta_{1}=\zeta_{2}=2 \%$ for comparison with the literature ${ }^{(5)}$ and for validation of the computer program. Although a slight mismatch is recognized among natural periods, as the model discretisation is rough, it can be found that the assumed model successfully reproduced the dynamic characteristics in the literature.

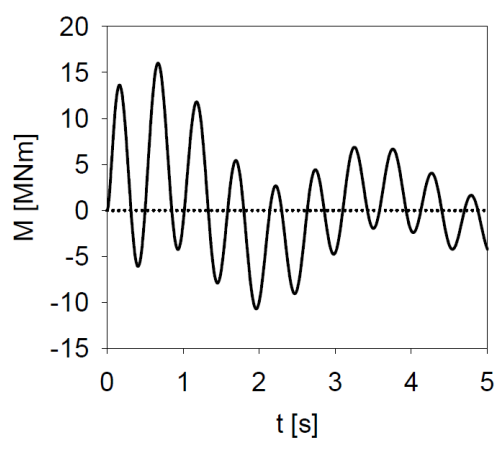

(a) Wienke ${ }^{(5)}$

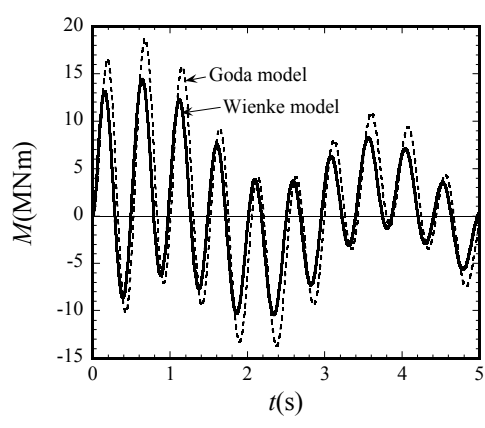

(b) Present calculation

Fig. 4 Time series of base overturning moments due to breaking wave impact force $\left(\lambda \eta_{\mathrm{c}}=1 \mathrm{~m}\right.$, included up to the $2^{\text {nd }}$ mode, $\left.\zeta_{1}=\zeta_{2}=2 \%\right)$ 
In order to compare the Goda model and Wienke model, Fig.5 demonstrates the maximum section forces at the sea bed, which are obtained by time dependent analysis. It can be found that the higher the mode, the lower the contribution. In the figure, the broken lines show the maximum section forces for each of vibration mode where the characteristics in Fig. 1 are clearly evident, i.e. for the first and second mode the Goda model value is larger than the Wienke model value but for higher modes the latter is almost twice the former. However, for the accumulated forces, which are indicated by the solid lines, these intrinsic dynamic characteristics are not seen clearly and even seem to be inconsistent, i.e. although the Goda model value is larger than Wienke model value up to the second modes for higher modes the relationship is reversed with respect to the overturning moment. This is because, in overturning moment, the higher mode contribution is relatively small compared with the base shear. As can be seen in Fig.5(c), this difference increases with damping ratio.

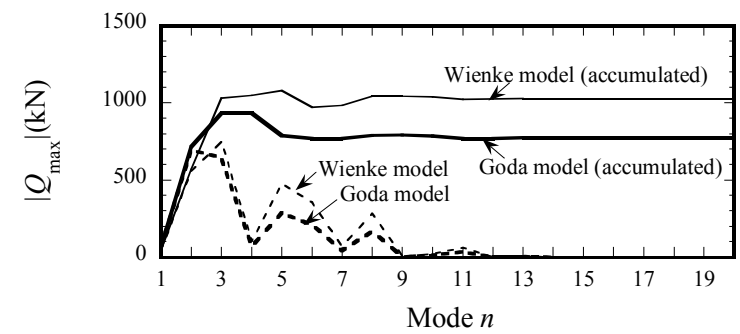

(a) Base shear, $\zeta_{1}=\zeta_{2}=2.0 \%$

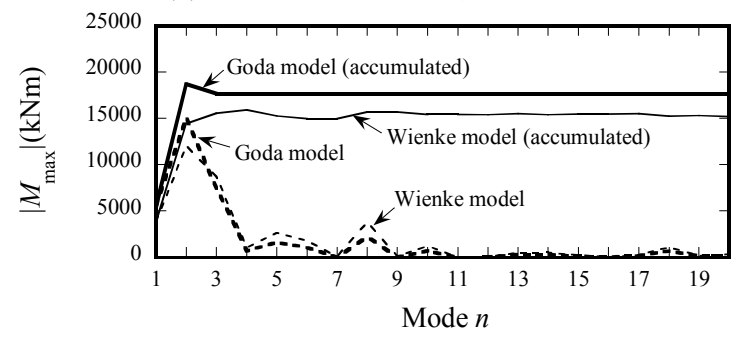

(b) Base overturning moment, $\zeta_{1}=\zeta_{2}=2.0 \%$

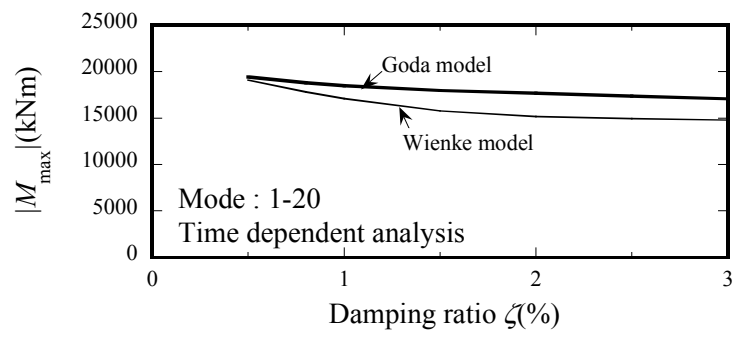

(c) Base overturning moment according to structural damping

Fig. 5 The maximum section forces by time dependent analysis $\left(\lambda \eta_{\mathrm{c}}=1 \mathrm{~m}\right)$

\subsection{Predictability of the maximum section forces by SRSS method}

Figure 6 compares the proposed method with time dependent analyses. For $\zeta=0.8 \%$, the proposed method with SRSS approximation provides reasonably conservative estimates if at least eight modes from the lowest is considered in Eq.(9) and Eq.(10).

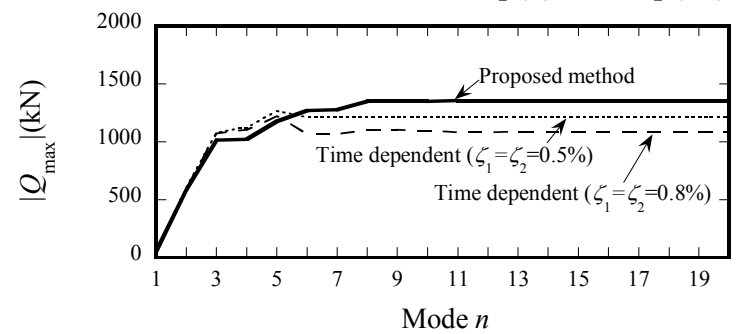

(a) Base shear 


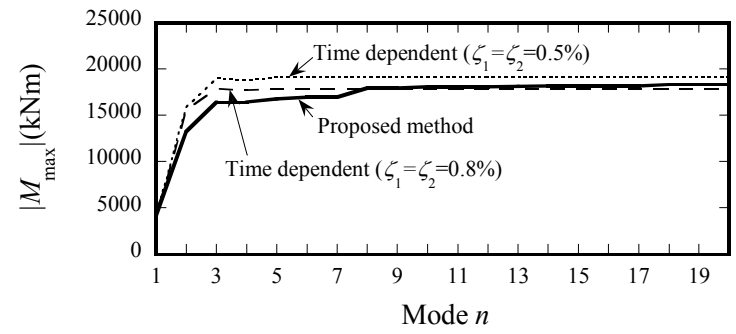

(b) Base overturning moment

Fig. 6 Comparison between the proposed method and time dependent analyses (Wienke model, $\left.\lambda \eta_{\mathrm{c}}=1 \mathrm{~m}\right)$

\subsection{Curling factor}

Batejes $^{(6)}$ has classified the breaker types into three categories as summarized in Table 2 in terms of surf similarity parameter $\xi_{b}=\tan \theta / \sqrt{H_{b} / L_{0}}$ where $\tan \theta$ is sea bed slope, $H_{b}$ is wave height at breaking point and $L_{0}$ is wave length. In the plunging wave region, breaking wave impact forces becomes the most important. Figure7 shows a diagram comparing the surf similarity parameter with the curling factor, which is established based on experimental studies ${ }^{(2)(7)}$. The hatched area in the figure corresponds to the region of the plunging wave, in which the curling factor increases drastically from the spilling wave regime and consequently a larger breaking wave impact force is expected. By considering the following assumptions, i.e. breaking wave impact force is less significant in the collapsing/surging region and the curling factor manifests its peak at the mid of the plunging wave region, the curling factor is approximated as :

$$
\lambda=\frac{\lambda_{\max }}{a\left\{\log \left(\xi_{\mathrm{b}} / \xi_{\mathrm{b}_{\max }}\right)\right\}^{2}+1}
$$

where, $\lambda_{\max }$ is the largest value of $\lambda, \xi_{\mathrm{b}_{\max }}$ is a surf similarity parameter corresponding to $\lambda_{\max }$ and $a$ is a parameter that determines the shape of the approximation. In Fig.7, $\lambda_{\max }=0.6, \xi_{\mathrm{b}_{\max }}=0.9$ and $a=4$ and 10 are demonstrated.

Table 2. Classification of breakers ${ }^{(6)}$.

\begin{tabular}{|c|c|c|c|}
\hline & Spilling & Plunging & Collapsing / Surging \\
\hline$\xi_{\mathrm{b}}$ & $\xi_{\mathrm{b}}<0.4$ & $0.4 \leq \xi_{\mathrm{b}}<2$ & $2<\xi_{\mathrm{b}}$ \\
\hline
\end{tabular}

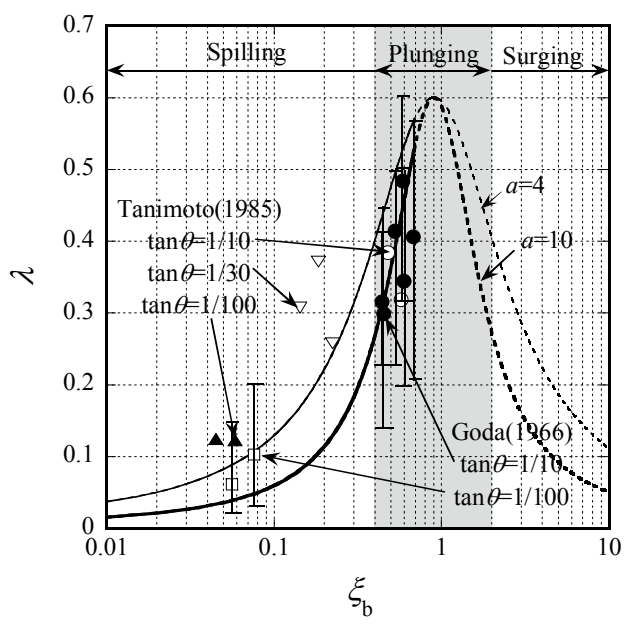

Fig. 7 Relationship between surf similarity parameter $\xi_{\mathrm{b}}$ and curling factor $\lambda$

Since no data are available from the middle of the plunging wave region to surging wave region, it is hoped that Eq.(16) will be updated by the accumulation of data in future studies. 


\section{Conclusions}

The dynamic response due to breaking wave impact force on an offshore wind turbine support structure was investigated. The Goda model and the Wienke model were compared, which are based on the von Karman and Wagner approximations. Furthermore, a simplified prediction procedure was proposed for high-frequency components in a breaking wave impact load acting on a pile-like support structure. The results of the study are summarized as follows :

(1) Impulse response factor of Goda model and Wienke model intersect at $\Omega_{j} \approx 4.72$, i.e. when $\Omega_{j}<4.72$, the former is larger than the latter. However when $\Omega_{j}>4.72$, it is opposite.

(2) When considering higher modes, the two models show different tendencies, i.e. the Wienke model shows larger value than the Goda model with respect to base shear, and the latter shows larger value than the former with respect to base overturning moment where the higher mode effect is less significant than lower mode effects.

(3) By the SRSS method, for damping factors above $0.8 \%$, by incorporating at least eight modes from the lowest, the maximum load effects by breaking wave impact force could be reasonably predicted on the conservative side.

(4) An approximation formula of curling factor was proposed as a function of surf similarity parameter.

Research following this paper, should be investigate through a number of case studies how much these breaking wave impact forces contribute to the total wave force. If the results of such investigation show that its contribution is not negligible and requires even further improvement, a Bagnold-type model might be a candidates for a more elaborate model of breaking wave impact force.

\section{References}

(1) Goda, Y., "Wave force on a vertical circular cylinder, Experiments and proposed method of wave force computation", Report of the Port and Harbour Research Institute, No.8(1964).

(2) Y. Goda et al., Study of impulsive breaking wave forces on piles, Report of Port and Harbour Technical Research Institute, Vol.6, No.5(1966), pp. 1-30.

(3) J. Wienke and H. Omneraci, Breaking wave impact force on a vertical and inclined slender pile - theoretical and large-scale model investigations, Coastal Engineering, 52(2005), pp.435-462.

(4) IEC : IEC61400-3 Ed.1 : Wind turbines - Part 3 : Design requirements for offshore wind turbines, 2009.

(5) J. Wienke et al., Theoretical formulation for wave slamming loads on a slender circular cylinders and application for support structures of wind turbines, 29th International Conference Coastal Engineering, ASCE, (2004), pp.4018-4026.

(6) Battjes, J.A., Surf similarity, Proc. of 14th Coastal Engineering Conference, (1974), pp.466-479.

(7) Tanimoto, K. et al., Experimental study on impulsive forces by breaking waves on circular cylinder, Report of the Port and Harbour Research Institute, Vol.25, No.2,(1986), pp.29-87. 